\title{
BMJ Open Establishing a population-based patient-reported outcomes study (PROMs) using national cancer registries across two jurisdictions: the Prostate Cancer Treatment, your experience (PiCTure) study
}

\author{
F J Drummond, ${ }^{1}{ }^{\mathrm{H}}$ Kinnear, ${ }^{2} \mathrm{C}$ Donnelly, ${ }^{2} \mathrm{E}$ O'Leary, ${ }^{1} \mathrm{~K}$ O'Brien, ${ }^{1} \mathrm{R} \mathrm{M}$ Burns, ${ }^{3}$ \\ A Gavin, ${ }^{2}$ L Sharp ${ }^{1}$
}

To cite: Drummond FJ, Kinnear H, Donnelly C, et al. Establishing a populationbased patient-reported outcomes study (PROMs) using national cancer registries across two jurisdictions: the Prostate Cancer Treatment, your experience (PiCTure) study. BMJ Open 2015;5:e006851. doi:10.1136/bmjopen-2014006851

- Prepublication history for this paper is available online. To view these files please visit the journal online (http://dx.doi.org/10.1136/ bmjopen-2014-006851).

$A G$ and $L S$ are joint last authors.

Received 8 October 2014 Revised 17 February 2015 Accepted 20 March 2015

CrossMark

For numbered affiliations see end of article.

Correspondence to Dr FJ Drummond; f.drummond@ncri.ie

\section{ABSTRACT}

Objective: To establish an international patientreported outcomes (PROMs) study among prostate cancer survivors, up to 18 years postdiagnosis, in two countries with different healthcare systems and ethical frameworks.

Design: A cross-sectional, postal survey of prostate cancer survivors sampled and recruited via two population-based cancer registries. Healthcare professionals (HCPs) evaluated patients for eligibility to participate. Questionnaires contained validated instruments to assess health-related quality of life and psychological well-being, including QLQ-C30, QLQPR25, EQ-5D-5L, 21-question Depression, Anxiety and Stress Scale (DASS-21) and the Decisional Regret Scale. Setting: Republic of Ireland (Rol) and Northern Ireland (NI).

Primary outcome measures: Registration completeness, predictors of eligibility and response, data missingness, unweighted and weighted PROMs.

Results: Prostate cancer registration was $80 \%(95 \% \mathrm{Cl}$ $75 \%$ to $84 \%$ ) and $91 \%$ (95\% Cl $89 \%$ to $93 \%$ ) complete 2 years postdiagnosis in $\mathrm{NI}$ and Rol, respectively. Of 12322 survivors sampled from registries, $53 \%(n=6559)$ were classified as eligible following HCP screening. In the multivariate analysis, significant predictors of eligibility were: being $\leq 59$ years of age at diagnosis $(p<0.001)$, short-term survivor ( $<5$ years postdiagnosis; $p<0.001)$ and from Rol $(p<0.001) .3348$ completed the questionnaire, yielding a $54 \%$ adjusted response rate. $13 \%$ of men or their families called the study freephone with queries for assistance with questionnaire completion or to talk about their experience. Significant predictors of response in multivariate analysis were: being $\leq 59$ years at diagnosis $(p<0.001)$ and from Rol $(p=0.016)$. Mean number of missing questions in validated instruments ranged from 0.12 (SD 0.71; EQ-5D-5L) to 3.72 (SD 6.30; QLQ-PR25). Weighted and unweighted mean EQ-5D-5L, QLQ-C30 and QLQ-PR25 scores were similar, as were the weighted and unweighted prevalences of depression, anxiety and distress.

\section{Strengths and limitations of this study}

- Both cancer registries used in this study are national, population-based and judged as high quality, and regularly collect vital status information.

- However, using cancer registries as the sampling frame meant that baseline and normative $\mathrm{HRQoL}$ and psychological well-being data were not collected.

- Validated questionnaires were used to measure health-related quality of life (HRQoL), utility, psychological well-being, and decisional regret to facilitate comparisons with other prostate cancer survivor studies and other diseases, and inform policy regarding survivor needs and services.

- Although questionnaires were developed with patient and clinical input, shorter questionnaires with fewer questions might increase the response rate and/or reduce data missingness. Data missingness should be addressed in analysis by using appropriate methods.

- The study design was cross-sectional, but a high proportion indicated a willingness to participate in follow-up research.

- While the response rate was $54 \%$, those who were deemed ineligible and non-responders may have different patient-reported outcomes (PROMs) than those who were eligible and responded. Weighting PROMs to the survivor population was undertaken to minimise the impact of these differences.

Conclusions: It was feasible to perform PROMs studies across jurisdictions, using cancer registries as sampling frames; we amassed one of the largest, international, population-based data set of prostate cancer survivors. We highlight improvements which could inform future PROMs studies, including utilising general practitioners to assess eligibility and providing a freephone service. 


\section{INTRODUCTION}

Prostate cancer is the most commonly diagnosed malignancy in men in developed countries. ${ }^{1}$ As in many countries, incidence rates in the Republic of Ireland (RoI) and Northern Ireland (NI) have increased significantly in the past two decades. ${ }^{2}{ }^{3}$ RoI was estimated to have had the highest prostate cancer incidence in Europe in 2008, while NI had the seventh highest incidence. ${ }^{4}$ This difference was due, in part, to a twofold higher rate of prostate-specific antigen (PSA) testing and prostate biopsies in RoI compared with NI. ${ }^{5}$ This in turn was driven partly by differences in health systems between jurisdictions; RoI has a mixed public/private healthcare system and NI has a predominantly public healthcare system.

Rising incidence, lower stage at detection and improving survival means that prostate cancer is the most prevalent male cancer. Additionally, the majority of men diagnosed with prostate cancer live for $\geq 5$ years. ${ }^{6}$ Indeed, in the USA, approximately $43 \%$ of all male cancer survivors have had prostate cancer. ${ }^{7}$ Consequently, over the past decade, there has been a growing interest in cancer survivorship research, that is, on the lived experiences and outcomes, including health-related quality of life (HRQoL), of those diagnosed with and treated for cancer. HRQoL is a multifaceted construct encompassing symptom burden, and physical and psychological functioning, ${ }^{8}$ and is increasingly being used as an end point in assessing treatment effectiveness. Despite this, there remains a paucity of population-based information on HRQoL, experiences, needs and other outcomes of short-term and long-term prostate cancer survivors. Lack of population-based information makes it difficult to determine the morbidity burden associated with prostate cancer, and its impact on men and their families. Furthermore, it complicates international comparisons of disease burden and the impact of alternative screening, diagnostic and management strategies. It also hinders policy-making and development of effective support services.

Patient-reported outcomes (PROMs), without clinical interpretation, are the optimum HRQoL measures. However, it can be difficult to recruit cancer survivors to PROMs studies. ${ }^{9}$ Cancer registries are increasingly being recognised as resources for PROMs and for other cancer survivorship studies, as sampling frames ${ }^{10-13}$ or as sources of clinical information when linked to other data sets. ${ }^{14}$ To date, relatively few national and even fewer international population-based PROMs studies have been conducted, and many do not include older and/or very long-term survivors. ${ }^{15}$

Our objective was to perform an international population-based PROMs study from among short-term ( $<5$ years), long-term (5-9.9 years) and very long-term ( $\geq 10$ years postdiagnosis) prostate cancer survivors, ${ }^{16}$ identified through cancer registries in two countries. We report on our experience of designing and conducting the study, and on HRQoL measures. We also describe recommendations to inform future research.

\section{METHODS}

\section{Health systems}

The island of Ireland comprises two jurisdictions, NI (part of the UK) and the RoI. RoI has a mixed public/ private healthcare system. Approximately $45 \%$ of the population have private health insurance, $30 \%$ have medical cards entitling them to free healthcare in the public system, and the other public patients make copayments for public health services. NI has a primarily public healthcare system, free at the point of use and funded through taxation, similar to the National Health Service in other parts of the UK.

Establishing an international population-based PROMs study among prostate cancer survivors involved a number of steps; (1) securing ethical approval; (2) identifying potentially eligible participants from cancer registries; (3) assessing participant eligibility; (4) questionnaire design; (5) identifying methods to maximise survey response and (6) linkage of questionnaire and cancer registry data.

\section{Ethical approval}

Research governance approval was obtained from each of the five NI Trusts. The study was conducted in accordance with the Declaration of Helsinki, ${ }^{17}$ and survivors consented to participate by returning completed questionnaires and/or signed consent forms.

\section{Identification of participants from national cancer registries in Rol and $\mathrm{NI}$}

The island of Ireland has two population-based cancer registries, one each in RoI (National Cancer Registry Ireland (NCRI)) and NI (Northern Ireland Cancer Registry (NICR)), which have registered all cancer cases since 1994 and 1993, respectively. Survivors were defined as men registered with invasive prostate cancer (International Classification of Diseases (ICD)10 C61), diagnosed between 1 January 1995 and 31 March 2010, and alive in November 2011. These limits were established to allow for maximum registration of men with prostate cancer and to ensure that the men had completed primary treatment(s) before being approached. In total, 22823 survivors were identified $(\mathrm{n}=17304$ (RoI); 5519 (NI)). A country-stratified random sample of 12322 men (54\% of total; $\mathrm{n}=7422$ (RoI); $\mathrm{n}=4900$ (NI)) was selected, with roughly equal numbers of $<5$ and $\geq 5$ years postdiagnosis.

\section{Assessment of survivor eligibility}

The same methods of recruitment were used in both jurisdictions, with modest refinements for local and/or national circumstances. In RoI and NI, similar to most European countries ${ }^{15}$ cancer registries do not have a mandate to contact patients with cancer directly without obtaining explicit clinician consent or agreement. This is also a requirement of ethical committees and research governance within RoI and NI. Therefore, survivors were screened for eligibility by healthcare providers (HCPs); 
general practitioners (GPs; $n=1745)$ in RoI and urology clinical nurses $(n=5)$ in NI. HCPs received a covering letter detailing the study and a form for each potentially eligible patient, which they were invited to complete and return. These were dispatched by post to GPs and freepost-addressed envelopes were supplied. Nonresponding GPs received up to two written reminders at two-weekly intervals, followed by a telephone follow-up. In NI, patient forms were dispatched by email. A follow-up email was sent after 2 weeks, followed by a telephone call after a further 2 weeks, if required. HCPs were asked to indicate whether or not each man was eligible to participate according to the criteria: the men were (1) alive, (2) aware of their prostate cancer diagnosis, (3) otherwise well enough to receive and complete a questionnaire (in particular, did not have a cognitive impairment), (4) able to understand English and (5) usually resident in RoI or NI.

\section{Survey instrument design and content}

Survivors were invited to complete a postal survey at one time point. A core questionnaire was developed from literature review and consultation with prostate cancer survivors and clinicians. To facilitate international comparisons, validated instruments were incorporated.

The core questionnaire included questions related to health at diagnosis; urinary (increased frequency, pain while urinating, blood in urine) or sexual symptoms (impotence/erectile dysfunction); comorbidities (heart or lung disease, stroke, diabetes, depression, high blood pressure, diverticular disease, bowel problems (constipation or diarrhoea)); PSA testing (and reasons for this); method of diagnosis (biopsy or transurethral resection of the prostate); and family history of prostate cancer.

Men were asked to identify primary treatment(s) received from a list (radical prostatectomy, external beam radiotherapy, androgen deprivation therapy (ADT), brachytherapy, chemotherapy, active surveillance/watchful waiting) with start and completion dates, and hospital (s) attended. They were also asked to identify any/all adverse effects experienced from a list (incontinence, impotence, loss of sexual desire, bowel problems (diarrhoea/constipation), gynaecomastia, hot flashes/flushes or sweats, fatigue, depression, other) with duration (number of months/ongoing at questionnaire completion) and any intervention(s) received to alleviate symptoms. Severity of adverse effects was explored using a five-point Likert scale (very mild (1) to very severe (5)) and men rated adverse effects as (1) the same, (2) not as bad or (3) worse than expected.

General and prostate cancer-specific HRQoL were measured using EORTC QLQ-C30 ${ }^{18}$ and QLQ-PR25 ${ }^{19}$ instruments, respectively. The EuroQoL EQ-5D-5L was included to measure utility. ${ }^{20}$ The 21-question Depression, Anxiety and Stress Scale (DASS-21) was employed to evaluate psychological well-being. ${ }^{21}$ Treatment decision-making and decisional regret were measured using the Control
Preference Scale, modified for the study, and Decision Regret Scale (DRS), respectively. ${ }^{22} 23$

Sociodemographic questions included age at diagnosis, marital and educational status, and men were asked if they would participate in future studies. Questions related to employment at diagnosis and questionnaire completion, productivity, financial stress and strain had been experienced previously. ${ }^{24} 25$

Both questionnaires were 28 pages long; the RoI version included 152 questions and the NI version contained 142 questions. The additional questions in the RoI questionnaire related to out-of-pockets costs incurred during diagnosis, staging, treatment and follow-up not included in the NI questionnaire. Order of the questions was largely the same in both questionnaires.

\section{Methods to maximise survey response}

Dillman's Tailored Design Method, and methods that have previously demonstrated an increase in response were used to maximise questionnaire response. ${ }^{26}{ }^{27} \mathrm{Men}$ were also provided with freephone number(s), one in each jurisdiction, with instructions (on questionnaires and information leaflets) to call if they required help completing the questionnaire. Additionally, it was branded as the PiCTure (Prostate Cancer Treatment, your experience) study. ${ }^{26}$ Men in RoI were randomised to receive either conditional or unconditional modest financial incentives. ${ }^{28}$ No incentives were used in NI.

\section{Linkage of questionnaire and cancer registry data}

Questionnaire responses were linked with cancer registration data to obtain/confirm date of birth, date of diagnosis, clinical stage (Tumour-Lymph Node-Metastasis (TNM) classification, V.5) and Gleason grade (GG). Additional clinical stage and GG information for NI responders was sought, as completeness for these variables in the NICR was low for early years.

\section{Analysis}

Analyses were performed using STATA V.13.1 (StataCorp LP, 2013).

\section{Completeness of cancer registration}

Completeness of prostate cancer registration in each registry was estimated using the flow method. ${ }^{29}$ Completeness estimates were calculated for all ages combined and by age group for 2007-2010 with follow-up for deaths until 31 December 2012.

\section{Predictors of eligibility to participate and questionnaire response}

Survival phases were categorised as short-term $(<5)$, longterm (5-9.9) and very long-term survivors ( $\geq 10$ years postdiagnosis). ${ }^{16}$ Univariate logistic regression analysis was used to investigate potential predictors of eligibility: age at diagnosis $(\leq 59,60-69, \geq 70$ years), survival phase (short-term, long-term, very long-term survivors) and jurisdiction (RoI, NI). Area of residence was further 
investigated by subdividing jurisdiction into eight health board (HB) regions (RoI) and five Trusts (NI). Variables significant at the $5 \%$ level were considered for inclusion in multivariate models; two multivariate models were run, one with area of residence specified by jurisdiction and one with area of residence specified by HB/Trust. The same approach was taken to investigate predictors of response among survivors deemed eligible to receive questionnaires.

\section{Acceptability of questionnaire}

The proportion and mean number of missing questions for QLQ-C30, QLQ-PR25, EQ-5D-5L, DASS-21 and DRS were calculated, as a potential measure of acceptability. The number of men who did not answer any surveyspecific treatment and/or adverse effect questions was also calculated. Missingness was investigated by jurisdiction, age at diagnosis and survival period, using analysis of variance tests.

\section{Unweighted and weighted PROMs}

Using QLQ-C30 and QLQ-PR25, Global Health Status (GHS), function, general and disease-specific symptom scores were linearly transformed to values between 0 (lowest) and 100 (highest). ${ }^{18} 19$ Mean (SD) utility scores were calculated using a crosswalk algorithm to convert the EQ-5D-5L to the three-level version..$^{20} \mathrm{~A}$ weightedhealth score based on UK normative data was assigned to each individual in the absence of normative data for Ireland. Scores for depression, anxiety and (di)stress were calculated from the DASS-21. ${ }^{21}$ Men were classified as having depression, anxiety and/or distress if they scored $\geq 10, \geq 8$ and $\geq 15$, respectively. ${ }^{21}$

Using survey methods, weights were computed based on time since diagnosis, age at diagnosis and jurisdiction. These were applied to responses by survey respondents to generate weighted PROMs.

\section{RESULTS}

\section{Completeness of cancer registration}

At 2 years postdiagnosis, estimated registry completeness for prostate cancer was $80 \%$ (95\% CI $75 \%$ to $84 \%$ ) in NI and $91 \%(95 \%$ CI $89 \%$ to $93 \%)$ in RoI. This increased to $89 \%$ (95\% CI $85 \%$ to $93 \%$ ) and $94 \%(95 \%$ CI $92 \%$ to $95 \%$ ) in NI and RoI, respectively, at 3 years postdiagnosis. Completeness of case ascertainment was higher for younger ( $\leq 59$ years) compared with older ( $\geq 80$ years) age groups.

\section{Predictors of eligibility of prostate cancer survivors}

Of the random sample of 12322 prostate cancer survivors, just over half $(53 \%$; $n=6559$; figure 1 ) were classified as eligible, following the HCP screening. In multivariate analysis, men were significantly more likely to be deemed eligible if they were $\leq 59$ years of age at diagnosis, short-term survivors and from RoI (table 1). Within each jurisdiction, a significant difference in the proportion of eligible survivors was observed by area of residence.

Reasons for ineligibility and barriers encountered to eligibility screening are shown in figure 1 . Briefly, in RoI, despite receiving responses from $86 \%(1546 / 1745)$ of

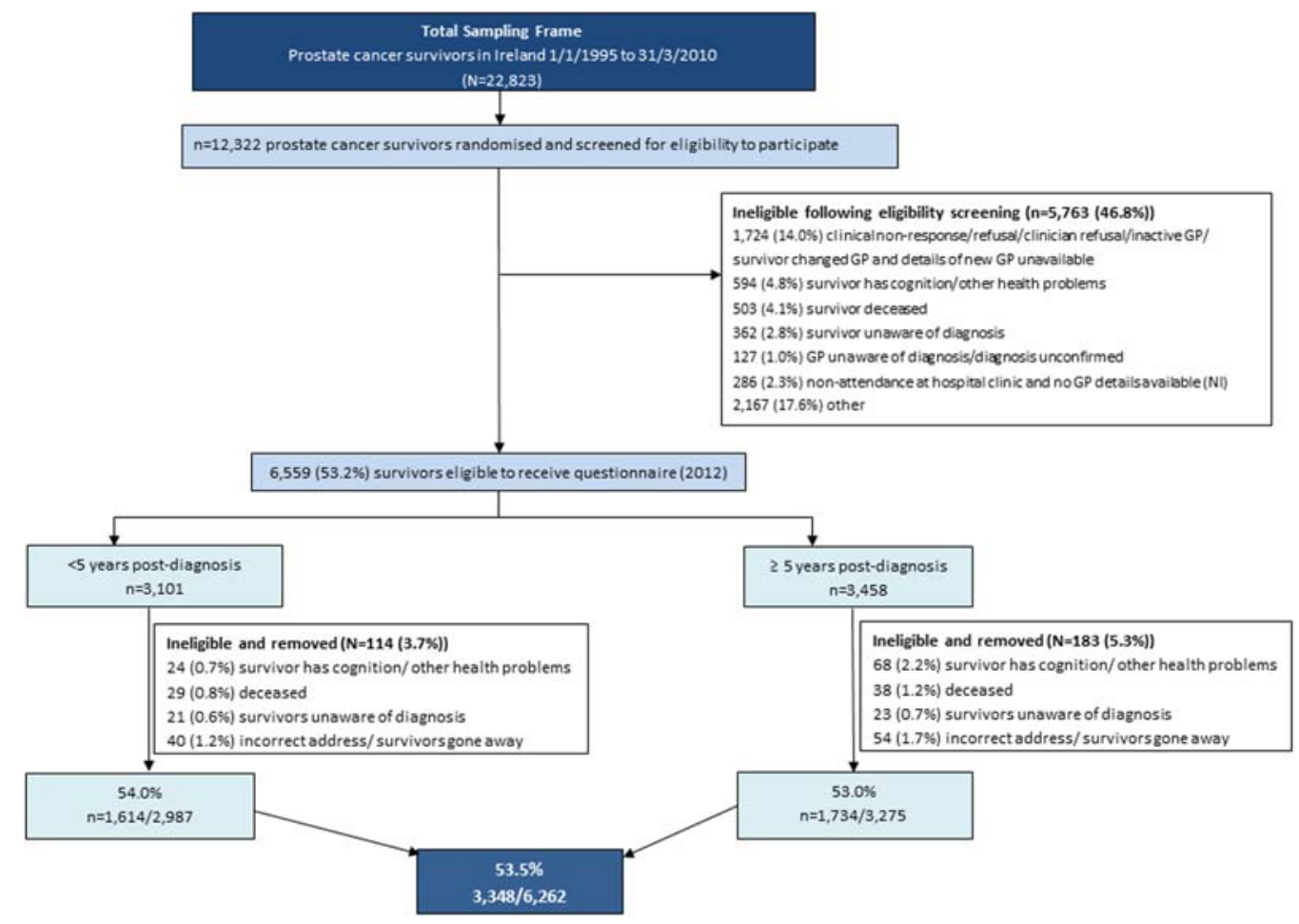

Figure 1 Flow diagram describing the recruitment of prostate cancer survivors (GP, general practitioner). 


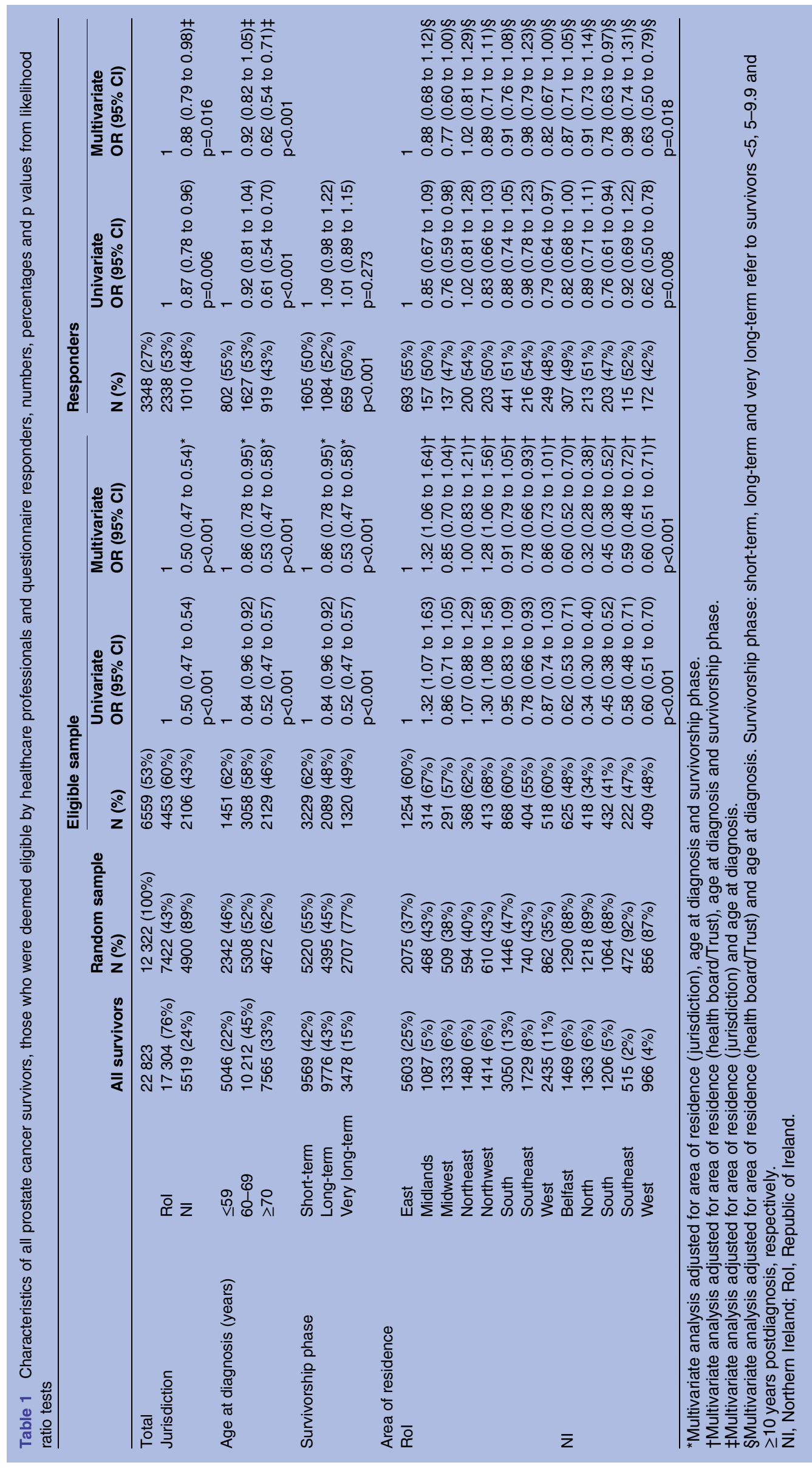


GPs contacted, $11 \%(827 / 7422)$ of survivors were lost due to GP non-response and $12 \%(897 / 7422)$ due to lack of up-to-date GP details, or GP's refusal to collaborate. A further $6 \%$ of RoI survivors were classified as ineligible $(400 / 7422)$ due to additional exclusion criteria imposed by GPs (eg, survivors were full-time carers or recently bereaved).

In NI, 6\% (286/4900) of the random sample had no recent contact with hospitals; thus, eligibility could not be checked. No reason was disclosed for ineligibility of $36 \%(1767 / 4900)$ of the NI sample.

Cognition impairments or other debilitating health problems accounted for 5\% (594/12 322) of the survivor sample being deemed ineligible $(\mathrm{n}=345$ (RoI), $\mathrm{n}=248$ (NI) ), 3\% (362/12 322) were considered unaware of their prostate cancer diagnosis $(\mathrm{n}=121(\mathrm{RoI}), \mathrm{n}=241(\mathrm{NI}))$, and $4 \%(503 / 12322)$ were recently deceased $(\mathrm{n}=341 \quad(\mathrm{RoI})$; $\mathrm{n}=162(\mathrm{NI})$ ). Overall, a lower proportion of survivors aged $\geq 70$ years at diagnosis were deemed eligible in NI compared with RoI (36\% and 52\%, respectively).

\section{Predictors of response}

Following questionnaire dispatch, 5\% (n=297) of potentially eligible survivors were discovered to be ineligible (eg, out-of-date address, ill health, recent death). After removing these from the denominator, the adjusted overall response rate was 54\% $(\mathrm{n}=3348 / 6263)$. In multivariate analysis, men from RoI and who were $\leq 59$ years of age at diagnosis were significantly more likely to respond than older men and those from NI (table 1). No significant difference in response was observed by time since diagnosis; $\geq 50 \%$ of men in each survival phase returned a questionnaire.

\section{Acceptability of questionnaire Missing data}

On average, $3 \%$ of questions designed to capture treatment(s) received and adverse effects experienced were unanswered (table 2). Overall, the proportion and mean number of missing questions varied across HRQoL and DRS instruments; the lowest was observed for EQ-5D-5L (0.22) and highest for QLQ-PR25 (2.94). In univariate analysis, mean number of missing questions was significantly associated with age at diagnosis for all sectionslowest for men $\leq 59$ years and highest for those $\geq 70$ years of age at diagnosis. Very long-term survivors had a significantly higher mean number of missing questions than long-term and short-term survivors for each instrument. Mean number of missing data for all instruments, except QLQ-PR25, was higher for RoI than NI respondents. Higher levels of missing data were found for later sections of the questionnaire.

\section{Freephone use}

Overall, 14\% ( $\mathrm{n}=906 / 6559)$ of all questionnaire recipients or their families called the freephone $(n=632$ (RoI); $\mathrm{n}=274(\mathrm{NI})$ ). The main reasons for calling were to inform us of their intention not to participate or provide reasons for non-participation (19\%; 173/906); administrative issues (eg, requesting a replacement questionnaire $(16 \% ; 149 / 906))$; queries regarding questionnaire content $(8 \% ; 76 / 906)$; being unaware of their prostate cancer diagnosis $(6 \% ; 50 / 906)$; or having data protection questions (eg, how their details were obtained; $2 \% ; 19 / 906)$. Sixty men $(7 \% ; 60 / 906)$ wanted to complete the questionnaire by telephone. Family members constituted 19\% (169/906) of calls; these calls were primarily to inform us that the questionnaire recipient was: too ill or distressed to participate, unaware of their prostate cancer diagnosis, or recently deceased. For $20 \%$ of callers $(n=177 / 906)$, the main reason for calling was to talk about their experiences, both good and bad, of living with prostate cancer.

\section{Willingness to participate in future research}

Almost three-quarters of respondents (overall: $\mathrm{n}=2423$ (72\%), n=1688 (72\%; RoI), n=735 (73\%; NI)) indicated that they would participate in future research.

Weighted and unweighted values for QoL, utility and psychological well-being measurements of respondents; generalisability to all prostate cancer survivors

Compared with all prostate cancer survivors in Ireland, long-term survivors were under-represented and survivors $>60$ years at diagnosis were over-represented among responders (table 3A). The overall mean unweighted utility score for participants was 0.82 (SD 0.22) and the GHS was 71.53 (SD 22.70; table 3B). Weighted and unweighted mean utility scores did not differ. Weighting resulted in lower GHS, physical, role and cognitive functioning mean scores, and lower general and diseasespecific symptom mean scores for all except appetite loss, constipation and ADT symptoms. Weighting also reduced the prevalence of depression, anxiety and distress.

\section{DISCUSSION}

We confirmed previous observations that cancer registries are appropriate sampling frames for PROMs studies ${ }^{15}$ and extend the existing evidence by demonstrating that international collaboration is feasible. To our knowledge, we have amassed the largest, population-based data set of prostate cancer survivors across two jurisdictions, which operate under different health systems, and have different legal and data protection environments. Using cancer registry data, we determined HRQoL, utility and prevalence of depression, anxiety and depression that have been weighted for all prostate cancer survivors in Ireland. Observations from this study could be used to optimise future PROMs research in cancer.

\section{Identification of potential participants from cancer registries}

Our aim was to be inclusive of all demographic groups, including older men, and we targeted those who were at 2 years or more postdiagnosis such that registration was 


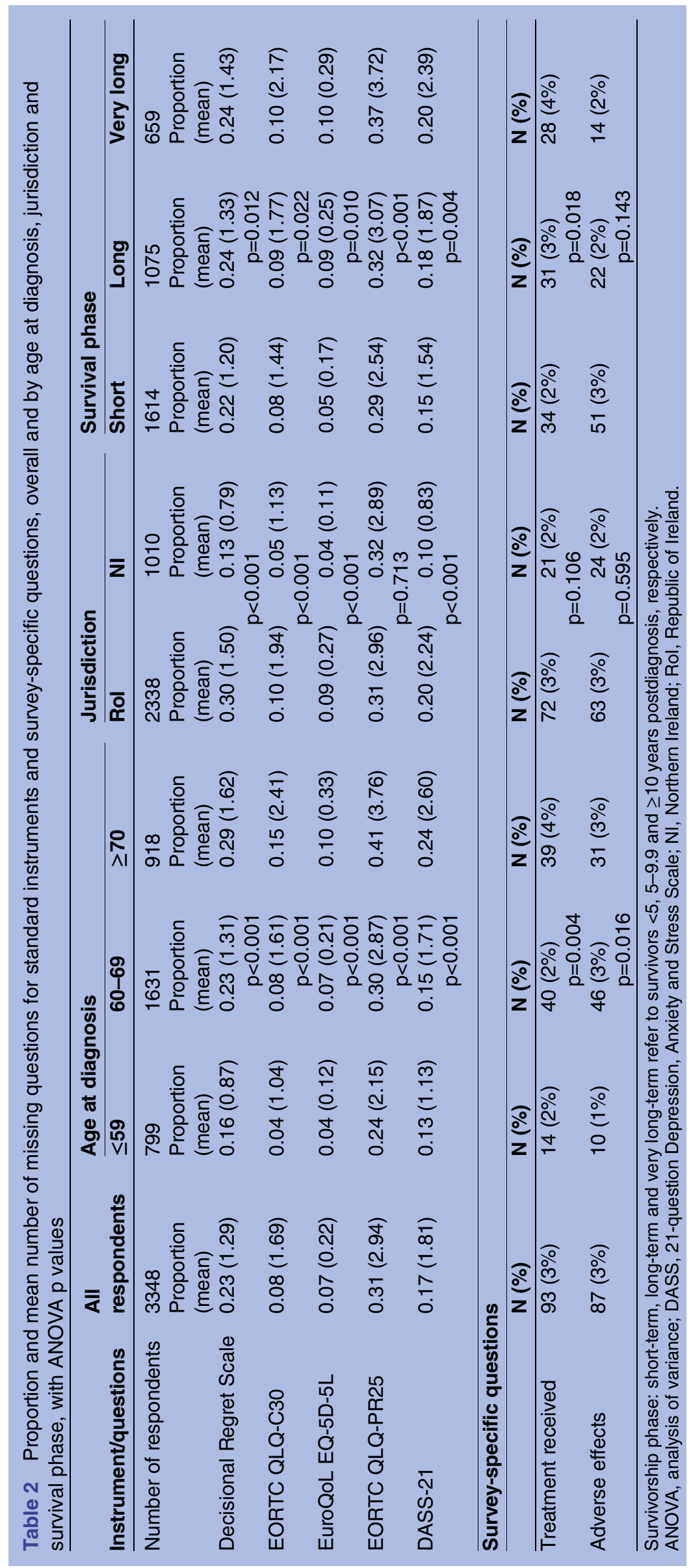


Table 3 Weighted and unweighted sociodemographic characteristics (\%) and; patient-reported outcomes (means and percentages) for PiCTure study respondents

\begin{tabular}{|c|c|c|c|}
\hline & & Unweighted \% & Weighted \%* \\
\hline \multicolumn{4}{|c|}{ Sociodemographic characteristics } \\
\hline \multirow{2}{*}{ Jurisdiction } & Rol & $70 \%$ & $77 \%$ \\
\hline & $\mathrm{NI}$ & $30 \%$ & $23 \%$ \\
\hline \multirow{3}{*}{ Age at diagnosis (years) } & $\leq 59$ & $24 \%$ & $22 \%$ \\
\hline & $60-69$ & $49 \%$ & $44 \%$ \\
\hline & $\geq 70$ & $24 \%$ & $34 \%$ \\
\hline \multirow[t]{3}{*}{ Survivorship phase } & Short-term & $48 \%$ & $42 \%$ \\
\hline & Long-tem & $32 \%$ & $43 \%$ \\
\hline & Very long-term & $20 \%$ & $16 \%$ \\
\hline \multicolumn{4}{|l|}{ Patient-reported outcomes } \\
\hline \multirow[t]{6}{*}{$E Q-5 D-5 L$} & Utility score & 0.82 & 0.82 \\
\hline & Mobility & 1.6 & 1.6 \\
\hline & Self-care & 1.2 & 1.2 \\
\hline & Usual activities & 1.6 & 1.7 \\
\hline & Pain/discomfort & 1.6 & 1.6 \\
\hline & Anxiety/depression & 1.4 & 1.4 \\
\hline EORTC QLQ-C30 & GHS & 71.53 & 71.15 \\
\hline \multirow[t]{5}{*}{ Functioning subscales } & Physical functioning & 82.16 & 81.54 \\
\hline & Role functioning & 80.91 & 80.57 \\
\hline & Emotional functioning & 83.51 & 83.66 \\
\hline & Cognitive functioning & 82.27 & 82.24 \\
\hline & Social functioning & 82.16 & 82.48 \\
\hline \multirow[t]{9}{*}{ Symptoms subscales } & Fatigue & 23.96 & 23.92 \\
\hline & Nausea and vomiting & 4.19 & 4.06 \\
\hline & Pain & 15.39 & 15.20 \\
\hline & Dyspnoea & 16.87 & 16.82 \\
\hline & Insomnia & 23.57 & 23.29 \\
\hline & Appetite loss & 7.00 & 7.00 \\
\hline & Constipation & 13.16 & 13.37 \\
\hline & Diarrhoea & 9.69 & 9.57 \\
\hline & Financial difficulties & 11.48 & 11.13 \\
\hline \multirow[t]{6}{*}{ EORTC QLQ-PR25 } & Urinary symptoms & 19.76 & 19.47 \\
\hline & Urinary bother & 15.57 & 14.79 \\
\hline & Bowel symptoms & 7.29 & 7.10 \\
\hline & Treatment symptoms & 10.62 & 10.17 \\
\hline & Sexual activity & 67.80 & 68.61 \\
\hline & Sexual functioning & 48.40 & 48.08 \\
\hline \multirow{3}{*}{ DASS-21 } & Depression & $16.5 \%$ & $16.3 \%$ \\
\hline & Anxiety & $16.4 \%$ & $16.1 \%$ \\
\hline & Distress & $10.6 \%$ & $10.4 \%$ \\
\hline
\end{tabular}

as complete as possible, and thus reduced possible sampling bias. Completeness of prostate cancer registration at 2 years postdiagnosis was lower than anticipated and varied between the registries, probably due to differences in registration processes, available data sources, rates of PSA testing and prostate biopsy, age and health of men at diagnosis and treatment patterns. ${ }^{35}$ Younger men and those undergoing surgery are likely to be registered before those managed by active surveillance or watchful waiting or ADT. As cancer registries are focused on completeness rather than timeliness of registration, such differences should be considered when designing and interpreting interjurisdiction studies, particularly studies aiming to recruit prostate cancer survivors at less than 2 years postdiagnosis.

\section{Assessment of survivor eligibility and ethical approval}

Despite collaborative and helpful HCPs, the need to screen men selected from registries for eligibility to participate meant that almost half of the randomised sample was deemed ineligible to participate after checks in the registries for recent deaths. In particular, some were excluded due to subjective reasons outside the specified exclusion criteria. Since this can affect population-basis 
data and generalisability of outcomes, effective methods are needed to optimise this process. Physician's notification rather than obtaining of physician's consent ${ }^{30}$ or contacting patients directly ${ }^{9}$ have been shown to be effective recruitment mechanisms which reduce these limitations. However, the latter would require changing cancer registry mandates, ethical and research governance processes despite research showing that most patients do not want physicians deciding whether they should be approached for study participation. ${ }^{31}$ Securing such changes in these data protection environments is likely to be challenging.

In RoI, a choice had to be made about assessing eligibility through hospital consultants or GPs. Using GPs offered several advantages, including (1) reducing the number of ethical applications required (from 28 to 1 , with time and cost savings), and (2) limiting the number of patients to be screened by individual HCPs ( $n=1-32$ (GPs); up to $\geq 1000$ (hospital-based HCPs). Within this study, our experience was that GPs in RoI excluded fewer patients and provided more explanations for exclusion than hospital-based HCPs in NI, possibly due to lengthy patient lists. However, we cannot discount the possibility that risk adversity in defining someone as eligible, on the part of nurses versus GPs, also contributed to the lower proportion of NI survivors being deemed eligible. A one-off study-specific modest monetary incentive to HCPs may improve participation and cooperation; ${ }^{32}$ performing additional data-finding exercises or implementing routine data-finding exercises to maintain up-to-date GP and contact information for patients within cancer registries could also help optimise eligibility processes. It is difficult, however, to identify methods to reduce the propensity of HCPs to exclude patients for non-study-specific reasons, other than to reiterate the eligibility criteria. Furthermore, while changes have been implemented to improve the effectiveness and uniformity of ethical applications in RoI, ${ }^{33}$ further improvements in ethical applications and research governance are still required.

\section{Response rate and item completeness; acceptability of the questionnaire to survivors}

Our overall response rate was similar to other studies using cancer registries as sampling frames. ${ }^{15}$ Participation among very long-term survivors was comparable to that from men diagnosed more recently and older men responded, albeit at a slightly lower response rate, than the younger men. This is a major strength of this study as older prostate cancer survivors, despite constituting the majority of survivors, are frequently omitted from PROMs studies. ${ }^{15}$ 34-37

The questionnaires used were long, which may have impacted on overall response. ${ }^{27}$ Although the RoI questionnaire included more questions than the NI version, the response rate was higher, which may be due to RoI survivors being slightly younger and/or use of incentives. ${ }^{28}$ However, questionnaires returned by RoI survivors had more missing data. Moderate levels of missingness are expected with PROMs instruments administered by post, the most common method of data collection for PROMs studies. People are also less likely to answer questions on sensitive topics and much of PROMs data collected, especially for prostate cancer, can be considered sensitive. The level of missingness from all survivors increased towards the end of the questionnaires, but this was more evident from the RoI responders, which may have been due to the higher number of questions and/ or the nature of these extra questions, which comprised detailed time, travel and out-of-pocket cost questions relating to prostate cancer diagnosis and treatment. Furthermore, the highest proportion of missing data from all survivors was observed for QLQ-PR25, which may relate to its structure and/or content. ${ }^{19}$ The EORTC QLQ-PR25 comprises 20 core and 5 conditional questions, organised into five multi-item subscales assessing: urinary, bowel and hormone treatment-related symptoms; sexual activity and sexual functioning (conditional on being sexually active); and a single conditional item assessing urinary bother due to use of incontinence aids. ${ }^{19}$ Most questions apply to the last week, and a few (relating to sexual functioning) to the previous 4 weeks. While the DASS and EORTC QLQ-C30 instruments did not contain conditional questions, their content could be considered sensitive by some, which may have contributed to relatively high proportions of missing data. Similarly, the structure of the DSR scale may have been misconstrued, with $89 \%$ answering one of the five questions, but only $58 \%$ answering all questions.

Therefore, strategies to increase response and reduce missingness are required. Future PROMs studies might consider using shorter questionnaires/including fewer questions, using alternative HRQoL instruments, ${ }^{38}$ and/or employing appropriate statistical methods to deal with data missingness. ${ }^{39}$

Methodologically, use of the freephone was successful in: facilitating response/participation (through providing clarifications and/or the opportunity to complete the questionnaire by telephone); eliciting reasons for non-response (including being unaware of their prostate cancer diagnosis); and identifying further gatekeeping issues. The volume of calls was unexpected and some men were looking for support and advice; however, few calls concerned data protection or unacceptability of questionnaires. Providing the freephone service put registry staff on the front line directly in contact with patients and had resource implication for the registries. Future researchers need to balance the advantages of a freephone with costs (including staff training and support) of providing the service.

\section{HRQoL and psychological health responders; generalisability to all prostate cancer survivors and international comparisons}

Despite significant differences between responders and all prostate cancer survivors within cancer registries, it was possible, from survey methodologies, to weight 
PROMs to make them more representative for all prostate cancer survivors. Compared with international studies, the overall HRQoL of prostate cancer survivors in Ireland, measured by the EQ-5D-5L utility score, was similar to that of short-term prostate cancer survivors in the UK, ${ }^{9}$ and the GHS and functional scores were higher than those of newly diagnosed, untreated men with prostate cancer in the EORTC reference population. ${ }^{40}$ The prevalence of depression and anxiety is slightly lower than that reported in a recent meta-analysis, ${ }^{41}$ while the prevalence of distress is similar to that previously described. ${ }^{42}$ Direct comparison of PROMs between studies is complicated by differences in eligibility criteria, data collection methods and response rates. To enhance comparability between studies over time, consideration might be given to standardising PROMs to internationally agreed external standards (as is routinely done for comparing cancer incidence rates).

\section{Strengths and limitations}

Both registries used in this study are national, population-based and judged to be of high quality by their inclusion in the Cancer Incidence in V Continents series of the International Agency for Research on Cancer, the cancer wing of WHO. However, using cancer registries as sampling frames meant we did not have baseline (ie, prediagnosis) HRQoL data as, by definition, registries do not record an individual's details until they have a cancer diagnosis. The study was crosssectional, collecting data at a single time point; however, the high level of willingness to participate in future research suggests that longitudinal data could be collected. PROMs are potentially sensitive to inaccuracies in recall, ${ }^{43}$ but are more valid measures of patient outcome than the clinician's judgement. Although we weighted PROMs by several key characteristics to make them more representative of all survivors, it is possible that eligible and ineligible survivors, and responders and nonresponders may have differed in other ways which would have impacted the outcome.

\section{Unanswered questions and future research}

We will exploit this data set to investigate factors associated with HRQoL and psychological well-being throughout survivorship, identify subgroups whose HRQoL has been adversely affected by prostate cancer and investigate morbidity burden weighted for all prostate cancer survivors. Data will inform cost-effectiveness models on PSA testing and mapping of PROMs data on the five-level EQ-5D-5L is planned. Findings will assist researchers and their clinical teams with treatment decision-making and the policymakers in determining the most efficient use of resources.

\section{CONCLUSION}

We have successfully used cancer registries, across two jurisdictions to amass a large data set of prostate cancer survivors, including older survivors and identified areas throughout the process which could be optimised in future PROMs research in cancer.

\section{Author affiliations}

${ }^{1}$ National Cancer Registry Ireland, Cork, Ireland

${ }^{2}$ Northern Ireland Cancer Registry, Centre for Public Health, Queen's University Belfast, Belfast, UK

${ }^{3} \mathrm{JE}$ Cairnes School of Business and Economics, National University of Ireland, Galway, Ireland

Acknowledgements The authors would like to thank all the healthcare professionals who helped to screen the survivors for eligibility and those who cooperated with us in obtaining ethical approval. They would like to thank Dr David Donnelly for statistical analysis performed during the course of this study, Dr Sandra Deady (NCRI) and Colin Fox (NICR) for data extraction, and Joanne Clooney (NCRI), Claire O'Callaghan (NCRI) and Audrey Craven-Lynn (NICR) for their work in survey administration and data entry. They would also like to thank Professor Ciaran O'Neill for intellectual input during the course of this study. Data entry was also undertaken by Patricia McDowell and Jonathan Mitchell in the NICR. They would like to thank the members of the Men Against Cancer (MAC) support group, all those who commented on the survey during development and those who pretested it. Finally, they would like to thank the men who completed the survey.

Contributors FJD drafted the manuscript, was involved in concept and design, securing funding, questionnaire design, and is the project manager Rol. HK was involved in concept and design, securing funding, questionnaire design, commented on the manuscript, and is the project manager NI. CD was involved in concept and design, securing funding, analysis, critical revision of the manuscript. EO'L was involved in statistical analysis. KO'B was involved in statistical analysis and critical revision of the manuscript. RMB was involved in critical revision of the manuscript. AG was involved in concept and design, securing funding, questionnaire design, critical revision of the manuscript, and is the principal investigator NI. LS was involved in concept and design, securing funding, questionnaire design, critical revision of the manuscript, and is the principal investigator Rol.

Funding This work was supported by Health Research Board in the Republic of Ireland (HRA_HSR/2010/17; FJD) and by Prostate Cancer UK (HK and FJD) and R\&D office of Public Health Agency in Northern Ireland (NI09-03; HK). Additional funding was provided by the National Cancer Control Programme (Rol; FJD and EO'L). The Northern Ireland Cancer Registry is funded by the Public Health Agency for Northern Ireland and the National Cancer Registry Ireland by the Department of Health.

Competing interests In 2011-2012, LS held an unrestricted project grant from Sanofi-Aventis to investigate treatment patterns in prostate cancer.

\section{Patient consent Obtained.}

Ethics approval Irish College of General Practitioners (Rol), Office for Ethics Committee Northern Ireland (ORECNI) (NI).

Provenance and peer review Not commissioned; externally peer reviewed.

Data sharing statement The questionnaire is available from the corresponding author-FJD, f.drummond@ncri.ie.

Open Access This is an Open Access article distributed in accordance with the Creative Commons Attribution Non Commercial (CC BY-NC 4.0) license, which permits others to distribute, remix, adapt, build upon this work noncommercially, and license their derivative works on different terms, provided the original work is properly cited and the use is non-commercial. See: http:// creativecommons.org/licenses/by-nc/4.0/

\section{REFERENCES}

1. Parkin DM, Bray FI, Devesa SS. Cancer burden in the year 2000 The global picture. Eur J Cancer 2001;37(Suppl 8):S4-S66.

2. Drummond FJ, Carsin AE, Sharp L, et al. Trends in prostate specific antigen testing in Ireland: lessons from a country without guidelines. Ir J Med Sci 2010;179:43-9. 
3. Donnelly DW, Gavin AT, Comber H. Cancer in Ireland 1994-2004: a comprehensive report. Northern Ireland Cancer Registry/National Cancer Registry Ireland; 2009. http://www.qub.ac.uk/researchcentres/nicr/FileStore/PDF/Filetoupload,185612,en.pdf

4. Jemal A, Centre MM, DeSantis C, et al. Global patterns of cancer incidence and mortality rates and trends. Cancer Epidemiol Biomarkers Prev 2010;19:1893-907.

5. Carsin AE, Drummond FJ, Black A, et al. Impact of PSA testing and prostatic biopsy on cancer incidence and mortality: comparative study between the Republic of Ireland and Northern Ireland. Cancer Causes Control 2010;21:1523-31.

6. De Angelis R, Sant M, Coleman MP, et al., EUROCARE-5 Working Group. Cancer survival in Europe 1999-2007 by country and age; results of EUROCARE-5-a population-bases study. Lancet Oncol 2014;15:23-34.

7. Siegel R, DeSantis C, Virgo K, et al. Cancer treatment and survivorship statistics, 2012. CA Cancer J Clin 2012;62:220-41.

8. Cella DF, Tulsky DS. Quality of life in cancer: definition, purpose, and method of measurement. Cancer Invest 1993;11:327-36.

9. Ganz PA, Land SR, Antonio C, et al. Cancer survivorship research: the challenge of recruiting adult long term cancer survivors from a cooperative clinical trials group. J Cancer Surviv 2009;3:137-47.

10. Glaser AW, Fraser LK, Corner J, et al. Patient-reported outcomes of cancer survivors in England 1-5 years after diagnosis: a crosssectional survey. BMJ Open 2013;3:e002317.

11. van de Poll-Franse LV, Horevoorts N, van Eenbergen M, et al., Profiles Registry Group. The Patient Reported Outcomes Following Initial treatment and Long term Evaluation of Survivorship registry: scope, rationale and design of an infrastructure for the study of physical and psychosocial outcomes in cancer survivorship cohorts. Eur J Cancer 2011;47:2188-94.

12. Garcia SF, Cella D, Clauser SB, et al. Standardizing patient-reported outcomes assessment in cancer clinical trials: a patient-reported outcomes measurement information system initiative. J Clin Oncol 2007;25:5106-12.

13. Bode RK, Hahn EA, DeVellis R, et al., Patient-Reported Outcomes Measurement Information System Social Domain Working Group. Measuring participation: the patient-reported outcomes measurement Information System experience. Arch Phys Med Rehabil 2010;91(9 Suppl):S60-5.

14. lowa Women's Health Study. Epidemiology of cancer in a cohort of older women. http://epi.grants.cancer.gov/Consortia/members/ iowawomen.html (accessed 29 Mar 2014).

15. Thong MS, Mols F, Stein KD, et al. Population-based cancer registries for quality-of-life research: a work-in-progress resource for survivorship studies? Cancer 2013;119(Suppl 11):2109-23.

16. American Cancer Society. Cancer facts and figures-2000. Atlanta: American Cancer Society, 2000.

17. WMA Declaration of Helsinki-ethical principles for medical research involving human subjects. 2008. http://www.wma.net/en/ 30publications/10policies/b3/17c.pdf (accessed 9 Jul 2014).

18. Aaronson NK, Ahmedzai S, Bergman B, et al. The European Organisation for Research and Treatment of Cancer QLQ-C30: a quality of life instrument for use in international clinical trials in oncology. J Natl Cancer Inst 1993;85:365-76.

19. van Andel G, Bottomley A, Fosså SD, et al. An international field study of the EORTC QLQ-PR25: a questionnaire for assessing the health-related quality of life of patients with prostate cancer. Eur $J$ Cancer 2008:44:2418-24.

20. Herdman M, Gudex C, Lloyd A, et al. Development and preliminary testing of the new five-level version of EQ-5D (EQ-5D-5L). Qual Life Res 2011;20:1727-36.

21. Lovibond SH, Lovibond PF. Manual for the Depression Anxiety Stress Scales. 2nd edn. Sydney: Psychology Foundation, 1995.

22. Lantz PM, Janz NK, Fagerlin A, et al. Satisfaction with surgery outcomes and the decision process in a population-based sample of women with breast cancer. Health Serv Res 2005;40:745-67.
23. O'Connor AM. User manual-Decision Regret Scale. Ottawa: Ottawa Hospital Research Institute, 1996:3. http://decisionaid.ohri. ca/docs/develop/User_Manuals/UM_Regret_Scale.pdf (accessed 22 Jan 2013).

24. Hanly P, Timmons A, Walsh PM, et al. Breast and prostate cancer productivity costs: a comparison of the human capital approach and the friction cost approach. Value Health 2012;15:429-36.

25. Sharp L, Carsin AE, Timmons A. Associations between cancer-related financial stress and strain and psychological well-being among individuals living with cancer. Psychooncology 2013;22:745-55.

26. Dillman DA. Mail and internet surveys. The tailored design method. 2nd edn. Wiley J and Sons Inc, 2007.

27. Edwards PJ, Roberts I, Clarke MJ, et al. Methods to increase response to postal and electronic questionnaires. Cochrane Database Syst Rev 2009;8:MR000008.

28. Drummond FJ, O'Leary E, Sharp L. Lotto more effect than prize draw in increasing response from a cancer patient postal questionnaire. J Clin Epidemiol 2015. In press, doi:10.1016/j. jclinepi.2015.01.01. Published Online First 27 Jan 2015.

29. Bullard J, Coleman MP, Robinson D, et al. Completeness of cancer registration: a new method for routine use. $\mathrm{Br} J$ Cancer 2000;82:1111-16

30. Smith T, Stein KD, Mehta CC, et al. The rationale, design, and implementation of the American Cancer Society's studies of cancer survivors. Cancer 2007;109:1-12.

31. Beskow LM, Sandler RS, Millikan RC, et al. Patient perspectives on research recruitment through cancer registries. Cancer Causes Control 2005;16:1171-5.

32. Drummond FJ, O'Leary E, O'Neill C, et al. "Bird in the hand" cash was more effective than prize draws in increasing physician questionnaire response. J Clin Epidemiol 2014;67:228-31.

33. HSE Research Ethics Committees Review Group 2008 Review of Research Ethics Committees \& Processes in Republic of Ireland. http://www.hse.ie/eng/services/Publications/corporate/etr/Review_of_ Research Ethics.pdf (accessed 26 Sep 2014).

34. Smith DP, King MT, Egger S, et al. Quality of life three years after diagnosis of localised prostate cancer: population based cohort study. BMJ 2009;339:b4817.

35. Johansson E, Steineck G, Holmberg L, et al., SPCG-4 Investigators. Long-term quality-of-life outcomes after radical prostatectomy or watchful waiting: the Scandinavian Prostate Cancer Group-4 randomised trial. Lancet Oncol 2011;12:891-9.

36. Mols F, van de Poll-Franse LV, Vingerhoets AJ, et al. Long-term quality of life among Dutch prostate cancer survivors: results of a population-based study. Cancer 2006;107:2186-96.

37. Gore JL, Kwan L, Lee SP, et al. Survivorship beyond convalescence: 48-month quality of life outcomes after treatment for localized prostate cancer. J Natl Cancer Inst 2009;101:888-92.

38. Rnic K, Linden W, Tudor I, et al. Measuring symptoms in localized prostate cancer: a systematic review of assessment instruments. Prostate Cancer Prostatic Dis 2013:16:111-22.

39. Bell ML, Fairclough DL. Practical and statistical issues in missing data for longitudinal patient-reported outcomes. Stat Methods Med Res 2014;23:440-59.

40. EORTC QLQ-C30 Reference values.0. http://groups.eortc.be/ HRQoL/sites/default/files/img/newsletter/reference values manual2008.pdf (accessed 22 Jan 2013).

41. Watts S, Leydon G, Birch B, et al. Depression and anxiety in prostate cancer: a systematic review and meta-analysis of prevalence rates. BMJ Open 2014;4:e003901.

42. Chambers SK, Zajdlewicz L, Youlden DR, et al. The validity of the distress thermometer in prostate cancer populations. Psychooncology 2014;23:195-203.

43. Rauscher GH, Johnson TP, Cho YI, et al. Accuracy of self-reported cancer-screening histories: a meta-analysis. Cancer Epidemiol Biomarkers Prev 2008;17:748-57. 